Archives de sciences sociales des religions

134 | avril - juin 2006

Varia

\title{
Le monde jéhoviste des imprimés
}

\section{Arnaud Blanchard}

\section{OpenEdition}

Journals

Édition électronique

URL : http://journals.openedition.org/assr/3396

DOI : 10.4000/assr.3396

ISSN : 1777-5825

Éditeur

Éditions de l'EHESS

Édition imprimée

Date de publication : 1 mai 2006

Pagination : 37-62

ISBN : 2-7132-2092-0

ISSN : 0335-5985

Référence électronique

Arnaud Blanchard, "Le monde jéhoviste des imprimés », Archives de sciences sociales des religions [En ligne], 134 | avril - juin 2006, mis en ligne le 11 juillet 2009, consulté le 01 mai 2019. URL : http:// journals.openedition.org/assr/3396 ; DOI : 10.4000/assr.3396 


\section{Arnaud Blanchard}

\section{Le monde jéhoviste des imprimés}

Le mouvement des Témoins de Jéhovah constitue un objet de recherche relativement désinvesti par les sciences sociales depuis plus d'une vingtaine d'années. L'intérêt pour cette formation a progressivement fléchi depuis la fin des années 1970 , époque à laquelle toute une série de travaux ${ }^{1}$ furent lancés. Cette mobilisation scientifique ne devait alors rien au hasard mais s'expliquait par une raison d'opportunité : les Témoins de Jéhovah offraient au milieu des années 1970 un terrain de vérification idéal des hypothèses de Leon Festinger sur les «prophéties manquées $»^{2}$. Les Témoins de Jéhovah fixant périodiquement des dates censées correspondre à l'ouverture du moment apocalyptique, et l'année 1975 ayant été mise en avant en ce sens, l'investigation sociologique visait, de manière latérale ou frontale, à comprendre quelles étaient les conséquences internes du dépassement de cette année. Mais, une fois passé ce moment historique, le suivi sociologique du groupe s'est distendu et les investigations de terrain se sont raréfiées.

Éclipsés à la fin des années 1970 par l'irruption des «nouveaux mouvements religieux $»^{3}$, dont l'efflorescence mettait en lumière les reconfigurations du paysage religieux contemporain, les Témoins de Jéhovah ont cessé de faire actualité pour la sociologie des religions. Et ce n'est que sporadiquement que l'organisation de la Tour de Garde réapparaît sur l'agenda scientifique à l'occasion de différends circonscrits, tel celui récurrent sur l'interdiction des transfusions sanguines ou

1. Social Compass, «Les Témoins de Jéhovah ", janvier 1977, vol. 24, n 1. James BECKFoRD, The Trumpet of Prophecy. A Sociological Study of Jehovah's Witnesses, Basil Blackwell, Oxford, 1975. Régis Dericquebourg, Les Témoins de Jéhovah. Dynamique d'un groupe religieux et rapport à l'institution. Essai de description psychosociologique d'une secte, thèse, EPHE, 1979.

2. Leon Festinger, Henry Riecken, Stanley SCHAchter, When Prophecy Fails: A Social and Psychological Study of a Modern Group that Predicted the Destruction of the World, New York, Harper \& Row Publishers, 1956. Sur la pertinence de cette analyse appliquée au cas des Témoins de Jéhovah, voir Mathew Schmalz, "When Festinger Fails: Prophecy and the Watch Tower », Religion, october 1994, vol. 24, no 4.

3. Pour une classification de ces groupes, se reporter à Danièle HERVIEU-LÉGER, « Le déferlement spirituel des nouveaux mouvements religieux », in Danièle HerviEu-LÉGER, Grace DAVIE, Identités religieuses en Europe, Paris, La Découverte, 1996. 
bien le contentieux qui l'oppose, en France, depuis quelques années à l'administration fiscale ${ }^{4}$. Cette relative atonie de la recherche signifie-t-elle, pour autant, que l'examen de ce mouvement religieux est désormais dénué d'enjeu ? Qu'y a-t-il encore à dire sur les Témoins de Jéhovah ?

L'entrée par les objets imprimés peut être ici féconde pour relancer l'imagination sociologique sur ce groupe et ouvrir de nouvelles voies d'exploration du monde jéhoviste, fort aujourd'hui de plus de six millions et demi de membres. Les imprimés forment en effet une réalité centrale dans le fonctionnement ordinaire de l'organisation dans la mesure où cette dernière est, historiquement et juridiquement, organisée autour d'une maison d'édition, la Watch Tower Bible and Tract Society ${ }^{5}$ (WTBTS). La raison sociale de cette société à but non lucratif, fondée dans les années 1880 aux États-Unis, est de produire et diffuser à travers le monde toute une variété d'imprimés (livres, magazines, brochures, tracts) afin d'informer la population de l'imminence de la fin de l'histoire humaine et de l'avènement du royaume de Dieu. Dans cette optique millénariste, des quantités considérables d'imprimés sont mises en circulation : à titre d'exemple, les deux titres de la presse jéhoviste, les hebdomadaires La Tour de Garde et Réveillezvous! sont tirés respectivement à vingt-cinq millions et à vingt-deux millions d'exemplaires, traduits dans des centaines de langues ${ }^{6}$. Plusieurs ouvrages publiés par la WTBTS connaissent, également, des taux de diffusion exceptionnels pour le secteur religieux, tel La vérité qui mène à la vie éternelle, traduit en plus de cent dix-sept langues et publié à plus de cent sept millions d'exemplaires entre 1968 et $1994^{7}$. Sous cet éclairage, l’organisation jéhoviste apparaît comme un empire de la littérature de piété, diffusant à flux réguliers des quantités industrielles d'imprimés.

De telles entreprises de presse ne sont pas exceptionnelles dans le monde croyant, et certaines ont retenu l'attention des historiens. Pour le catholicisme, le cas de l'abbé Migne ${ }^{8}$, éditeur au XIX ${ }^{e}$ siècle d'une monumentale Bibliothèque

4. Par un arrêt de la cour d'appel de Versailles en date du 28 février 2002, un redressement fiscal - d'un montant de plus de 45 millions d'euros - visant l'association des Témoins de Jéhovah a été confirmé. En cas d'exécution de la peine, les assises financières du jéhovisme français seraient considérablement fragilisées. Sur le détail du dossier, voir Michel DE GUILLENSCHMIDT, "La révélation des dons manuels ou l'apocalypse fiscale ", Revue de jurisprudence fiscale, décembre 2000. Pour un panorama des tensions entre le groupe et les pouvoirs publics en France, se reporter à Régis DERICQUeBourg, "Les Témoins de Jéhovah : vers une sortie de la logique sectaire ? ", in Françoise CHAMPION, Martine COHEN, dirs., Sectes et démocratie, Paris, Seuil, 1999.

5. La Tour de Garde, société de Bibles et de tracts. Les Témoins de Jéhovah sont également identifiés sous l'appellation "mouvement de la Tour de Garde ".

6. Ordres de grandeur annoncés par l'organisation pour l'année 2003.

7. Consulter Dominique Fremy, Michèle Fremy, Quid 2004, Paris, Robert Laffont, 2003, p. 361.

8. Howard Block, Le plagiaire de Dieu. La fabuleuse industrie de l'abbé Migne, Paris, Seuil, 1996. 
universelle du clergé, mérite ici d'être signalé, tout comme celui de Mgr Benigni ${ }^{9}$, défenseur intransigeant de la politique pontificale et instigateur, au début $\mathrm{du} \mathrm{Xx}^{\mathrm{e}}$ siècle, d'un réseau international d'information, la Sapinière. Mais dans ces deux cas, il s'agissait essentiellement d'entreprises éditoriales individuelles, s'adressant à des lecteurs finaux sans souci de les intégrer durablement à une action commune. Une toute autre configuration se présente avec la WTBTS dans la mesure où cette maison d'édition travaille, précisément, à construire une relation singulière (par son intensité morale) et pérenne avec ses lecteurs, à les organiser en groupes locaux et à les faire participer activement à la diffusion de ses imprimés.

D’un point de vue matériel, les Témoins de Jéhovah sont des individus abonnés, affiliés, à la WTBTS de laquelle ils reçoivent, sur une base régulière, toute une gamme d'imprimés destinés à des usages différenciés. Ces imprimés occupent une place centrale dans le quotidien des membres répartis en groupes locaux appelés "congrégations ». Lors des cinq heures de réunion hebdomadaire dans ces assemblées locales, plusieurs imprimés sont collectivement déchiffrés, organisant une sociabilité spécifique. De plus, l'acheminement des imprimés au grand public scelle une pratique distinctive des Témoins de Jéhovah : le porteà-porte à domicile. Tous les Témoins de Jéhovah consentent à cette forme de colportage, érigée en observance religieuse, à raison de quatre heures de démarchage, en moyenne, par semaine. Si un examen des activités concrètes des Témoins de Jéhovah tend, ainsi, à les présenter comme d'infatigables lecteurs et colporteurs, alors la circulation des imprimés peut être un enjeu suffisamment saillant pour organiser tout un ensemble de relations sociales (sociabilité interne, modalités du croire, fonctionnement quotidien de l'organisation). Dans ces conditions, le suivi des objets imprimés est susceptible de constituer un moyen heuristique efficace pour appréhender l'organisation tant de manière globale - à travers l'analyse du parcours historique du groupe - qu'en détails, en mettant au jour les fondements de l'expérience jéhoviste. Avant de mettre en pratique cette piste de recherche, il convient dans un premier temps de préciser comment les imprimés sont appréhendés sociologiquement.

\section{Un détour par la sociologie des imprimés}

Les travaux de sciences sociales consacrés aux objets imprimés reposent sur la distinction entre l'imprimé et le texte ${ }^{10}$. Les objets imprimés ne se réduisent

9. Émile Poulat, Catholicisme, démocratie et socialisme. Le mouvement catholique et Mgr Benigni, de la naissance du socialisme à la victoire du fascisme, Paris, Casterman, 1977.

10. Voir Roger CHARTIER, Culture écrite et société. L’ordre des livres (XIV ${ }^{e}-X V I I I^{e}$ siècles), Paris, Albin Michel, 1996. Et Roger CHARTIER, « Textes, imprimés, lectures », in Martine PoulaIN, dir., Pour une sociologie de la lecture. Lectures et lecteurs dans la France contemporaine, Paris, Éditions du Cercle de la Librairie, 1988. Pour une synthèse plus récente, se reporter à Gérard MAUGER, "Écrits, lecteurs, lectures », Genèses, mars 1999, nº 34. 
jamais aux textes; plutôt, les textes se donnent à lire dans des dispositifs formels et matériels qui les portent (par exemple, pour le livre imprimé, le format, les dispositions de la mise en page, le découpage du texte, les conventions réglant sa présentation typographique, etc.). Aussi, un texte est-il inséparable du support qui le propose au déchiffrement. De cette manière, l'imprimé est un objet destiné à la lecture ${ }^{11}$. En tant que tel, il est le produit d'un auteur mais aussi d'un éditeur qui propose un texte à la consultation en fonction, par exemple, des attentes et compétences supposées d'un lectorat. En tant que produit, l'imprimé suit une trajectoire pour rejoindre les lecteurs. Une fois confectionné, il transite dans des circuits de distribution pour atteindre des lecteurs, attendus ou inédits, sans induire nécessairement de leur part un mode de lecture.

En prêtant attention à la place structurante de l'imprimé dans le fonctionnement concret des Témoins de Jéhovah, on peut appréhender l'histoire de ce mouvement comme celle d'un travail éditorial multiforme (sur les matérialités des textes, leur rédaction, le mode de circulation qu'ils empruntent) réalisé par la WTBTS et son personnel afin de réduire la marge d'interprétation individuelle vis-à-vis des textes diffusés et de structurer à distance les activités des lecteurs. En effet, la maison d'édition est une société de production d'imprimés vecteurs d'une alerte spécifique. Cette alarme, relative à l'achèvement imminent de l'histoire humaine, engage l'avenir eschatologique de chacun et de tous. Pour fonctionner, c'est-à-dire pour être simultanément plausible et diffusée, reprise par d'autres, cette alarme doit pouvoir soutenir l'urgence et l'importance de la menace, et éviter que le destinataire ne se trompe sur la nature du message et ne le considère comme un pur dispositif informationnel ou, à l'inverse, comme une pure agitation argumentative.

Au cours de l'histoire du mouvement de la Tour de Garde, l'imprimé a été l'objet sur lequel se sont éprouvés, traduits, les impératifs de circulation et de décryptage adéquats de l'alerte millénariste. Autour des imprimés, et des conditions de leur consultation, s'est opérée une régulation des modes de lecture des textes avec l'organisation progressive d'une sociabilité spécialisée (des groupes formalisés de lecteurs), visant à laisser le moins de prise possible aux recompositions individuelles des textes d'alerte. Parallèlement, la mise en place d'un approvisionnement en imprimés particulier et contraignant (la production et la distribution en propre des imprimés, en recourant aux fidèles) a permis de fixer l'attachement des individus au système de diffusion de l'alarme apocalyptique. Afin de mettre à l'épreuve cet argument, la présentation est articulée autour de trois axes.

Dans un premier temps, l'histoire de l'organisation - de sa genèse dans les années 1880 jusqu'aux années 1940 - est restituée en mettant l'accent sur le

11. Cependant, un imprimé peut être consacré à autre chose qu'à la lecture, et être utilisé par exemple à des fins de cérémonie ou de dévotion. Voir notamment Alain BouREAU, « Adorations et dévorations franciscaines. Enjeux et usages des livrets hagiographiques », in Roger CHARTIER, dir., Les usages de l'imprimé (XV'-XIX ${ }^{e}$ siècles), Paris, Fayard, 1987. 
moment charnière que constituent les années 1920-1940. L'ensemble des travaux de sciences sociales convergent sur le fait que de profondes mutations (organisationnelles et doctrinales) se produisent pendant cette période au sein du groupe. Classiquement, ces transformations sont analysées en termes de basculement du type du réseau mystique à celui de la secte perçue comme association volontaire et sélective d'individus cultivant l'intensité de la vie religieuse. Le mouvement de la Tour de Garde, qui rassemble d'abord de manière lâche les sympathisants d'un écrivain millénariste, Charles Russell, se métamorphose, en une vingtaine d'années, en une centrale du croire hiérarchiquement organisée et reposant sur l'exclusivité de l'appartenance religieuse et la vigueur de la participation individuelle. La perspective développée ici reprend cette thèse mais déplace l'attention vers les objets imprimés et les rapports sociaux (de production, de distribution, de croyance) que leur circulation compose. De ce point de vue, les transformations affectant le mouvement pendant cette période sont non seulement descriptibles par la figure de l'imprimé, mais se réalisent socialement par sa médiation (changement des modes de production, de distribution, et de consultation de ces imprimés, eux-mêmes profondément remaniés sur le plan textuel).

S'installant dans la logique sociale de l'alarme, la maison d'édition entame alors, pendant ces années de transition, un travail social et éditorial consistant à organiser la filière suivie par l'imprimé (les conditions de production et de diffusion, les pratiques respectives des distributeurs et des lecteurs) de manière à faire circuler son message d'éveil millénariste sur une large échelle et à encadrer autant que possible son mode de réception. Ainsi, le second point d'argumentation est qu'à cette époque charnière, le mouvement prend la forme d'une centrale d'alarme, c'est-à-dire d'une organisation intégrée qui, non seulement émet des messages d'alerte et d'urgence par voie d'imprimés, mais se dote d'une structure interne (rapports de subordination des groupes locaux vis-à-vis du centre) et de mécanismes de transmission (par l'intermédiaire de dispositifs techniques et de ses membres) visant à diffuser les alertes en comprimant les possibilités de reformulation ou de détournement de sens.

Enfin, dans un troisième temps, un examen synchronique de l'organisation, telle qu'elle se présente actuellement (depuis les années 1940, à partir desquelles les caractéristiques socioreligieuses des Témoins de Jéhovah se stabilisent) est proposé. Les Témoins de Jéhovah peuvent être étudiés comme une communauté de lecteurs. Des conventions de lecture sont inculquées, partagées, et activées lors de la consultation des imprimés de la WTBTS en groupe ou individuellement, au domicile des fidèles. Ces façons de lire résultent tant du type de sociabilité nouée lors des séquences de lecture en groupe que des formes mêmes des imprimés, qui organisent un certain rapport entre le texte présenté et le lecteur, régulier ou occasionnel. Le vécu jéhoviste se construit ainsi à partir d'imprimés : il est, non seulement, participation à un réseau de distribution et de déchiffrement d'imprimés spécifiques, mais aussi, capacité à dire le croire et à le valider en congrégation par l'adhésion, la reprise littérale des énoncés jéhovistes publiés. 


\section{D'une mouvance éclatée de lecteurs à une centrale du croire}

Aux origines des Témoins de Jéhovah se trouve la nébuleuse des étudiants de la Bible, une mouvance qui émerge aux États-Unis à partir des années 1880 dans la lignée des enseignements d'un entrepreneur de presse, Charles Russell (1852-1916). Ce dernier, formé auprès d'écrivains et de prédicateurs adventistes, collabore à plusieurs journaux avant de lancer, avec quelques associés, son propre bulletin, Zion's Watch Tower and Herald of Christ's Presence, "Le phare de la Tour de Sion et messager de la présence du Christ ${ }^{12}$, et de fonder en 1881 une maison d'édition ${ }^{13}$.

L'ambition de Russell, et de ses proches, n'est pas de créer un nouveau mouvement religieux mais, simplement, de diffuser la Bible et de faire connaître la lecture particulière qu'ils en font. En effet, Ch. Russell est convaincu que les textes bibliques décrivent, selon un mode de narration et un codage à élucider, le devenir historique de l'humanité, de son origine à son terme. Source unique de connaissance de Dieu, la Bible est, ainsi, considérée comme un texte en partie crypté qu'il s'agit de scruter minutieusement afin de comprendre la manière dont la marche humaine y est écrite. Cette approche des textes sacrés repose sur la croyance en un "plan divin des âges ", selon le titre d'un des ouvrages à succès de Russell, c'est-à-dire sur la proposition que Dieu dispose d'un programme pour l'humanité, comprenant plusieurs étapes et menant inéluctablement vers un dénouement imminent. L'histoire humaine, ponctuée et sériée en plusieurs époques, tend à son achèvement et débouche vers la nouvelle phase régénératrice qu'est la réalisation historique du royaume de Dieu. De fait, inscrits dans une filiation adventiste et millénariste ${ }^{14}$, Charles Russell et ses proches produisent une littérature d'études bibliques - dans un style qui alterne gloses et commentaires savants avec des passages plus lyriques et poétiques - qui affirme l'arrivée de la concordance des temps historique et apocalyptique. Dans cet esprit, plusieurs années sont même annoncées à l'issue de calculs chronologiques sophistiqués, telle l'année 1914, censée inaugurer le règne visible de Jésus-Christ sur terre.

Le domaine d'action de Russell est avant tout éditorial et biblique : la maison d'édition qu'il dirige diffuse des exemplaires de la Bible, selon les traductions protestantes courantes, rachetées par lots à des maisons d'édition commerciales et revendues à bas prix, ainsi qu'une multitude de fascicules et de tracts présentant les enseignements chrétiens traditionnels (sur la théorie de l'évolution, le spiritisme,

12. Son titre change en 1909 pour devenir « La Tour de Garde et Messager de la Présence de Christ".

13. Il s'agit de la Zion's Watch Tower Society, rebaptisée en 1896 Watch Tower Bible and Tract Society.

14. Quelques pages sont consacrées à Russell dans Eugen Weber, Apocalypses et millénarismes. Prophéties, cultes et croyances millénaristes à travers les âges, Paris, Fayard, 1999, (p. 217-218), ainsi que dans Jean Delumeau, Mille ans de bonheur, Paris, Fayard, 1995, (p. 401-402). 
ou la prééminence de la Bible). À côté, et dans des matérialités distinctes (dans le magazine, dans des livres et des brochures dédiés), sont publiées les attentes millénaristes de Charles Russell et de ses associés. Ces différents écrits sortent de leur rédaction sous forme de tracts et d'opuscules, puis sont rassemblés au sein d'une collection de six livres, Studies in the Scriptures (Études des Écritures), série de référence qui connaît une diffusion massive. Le premier volume, «Le plan divin des âges ", est tiré à quatre millions trois cents mille exemplaires du vivant de Russell (jusqu'en 1916), et à plus de six millions vers 1930. Pour confectionner ses différents produits littéraires, la maison d'édition a recours à des imprimeries commerciales réparties sur le territoire américain ainsi qu’à l'étranger pour une diffusion en dehors des frontières. En outre, si le magazine est vendu par abonnement et par l'intermédiaire des services postaux, les brochures et les livres sont acheminés par un réseau original de colporteurs-missionnaires.

Dès 1881, la Watch Tower Bible and Tract Society passe dans son périodique une annonce d'embauche de mille colporteurs: durant les trente premières années, leur nombre varie entre une poignée et six cents environ. Pourtant leur travail est déterminant : en effet, la WTBTS renonce très tôt à distribuer ses ouvrages dans le réseau des librairies commerciales. Ses livres sont portés et vendus directement par des colporteurs chargés de les écouler. Ces derniers sont des quasi-bénévoles : remboursés de leur frais de transport, ils sont rémunérés sur la vente d'ouvrages et sur le nombre d'abonnements souscrits. Conjointement placiers et missionnaires, il leur revient de mettre en place des cellules de lecture des publications de la WTBTS, appelées "classes d'étudiants de la Bible ", et d'animer pour un temps ces dernières afin de diffuser l'approche russelienne des textes bibliques. Afin de consolider le réseau de ces classes d'étudiants, un autre programme, complémentaire à celui des colporteurs, est lancé en 1894 sous le nom de travail de pèlerin : les pèlerins sont des bénévoles voyageant à plein temps d'une localité à l'autre pour y coordonner des groupes de lecteurs. Hommes de confiance de Russell, ils ont la charge de faire appliquer les directives de la WTBTS (précisées plus loin) sur le terrain et de conduire des enseignements bibliques. De plus, sur des territoires encore inexplorés par les colporteurs (hors des ÉtatsUnis singulièrement), les pèlerins apportent des imprimés et y coordonnent les personnes sollicitées en classes d'étudiants de la Bible.

Dans une plus large mesure que les colporteurs, les pèlerins sont susceptibles de se conduire en entrepreneurs religieux indépendants au cours de leurs périples, revendiquant par exemple un rôle personnel dans le scénario millénariste. Bénéficiant des ressources de la WTBTS (fourniture en imprimés, remboursement de certains frais), les pèlerins en mission sont totalement autonomes dans la conduite de leur activité et dans l'organisation du réseau d'étudiants de la Bible. Nombre d'entre eux réunissent ainsi, à l'étranger, les moyens matériels nécessaires à la dissémination des publications de la WTBTS (imprimerie ou dépôt d'imprimés, enrôlement de bénévoles ou de colporteurs rémunérés). Par la suite, libres de 
toute contrainte de la part de la WTBTS, ils diffuseront leurs propres pensées soit en les intégrant aux imprimés traduits (ils sont les traducteurs locaux des écrits américains), soit en utilisant les capacités d'impression développées sur place ${ }^{15}$. Ces sécessions sont révélatrices du faible degré de structuration des lecteurs de Russell.

Le lectorat des publications de la WTBTS constitue pendant longtemps une nébuleuse, sporadiquement organisée et reliée de manière fluide à la maison d'édition. Démarchés par les pèlerins et les colporteurs, ou bien intéressés à l'action de Charles Russell par l'intermédiaire de techniques promotionnelles innovantes ${ }^{16}$, des lecteurs et des abonnés au magazine se regroupent en plusieurs endroits du territoire américain, sans consignes précises de la part de la WTBTS au départ. Pendant longtemps, les «classes d'étudiants de la Bible » seront caractérisées par leur hétérogénéité et leur indépendance vis-à-vis de la WTBTS. Ce qui rassemble, localement, ces étudiants est un intérêt commun pour le travail de Russell, intérêt qui oscille entre la simple curiosité intellectuelle et la conviction que celui-ci est bien le «serviteur fidèle et avisé » annoncé dans l'Évangile de Matthieu $(24,45)$, celui qui demeure vigilant dans l'attente du retour imminent du Christ. Aussi, la diversité est-elle la règle : les groupes s’administrent souverainement et déterminent, seuls, le type d'activités qu'ils mènent; le contenu et la périodicité des réunions varient ainsi d'une classe à l'autre. Le plus souvent, néanmoins, les participants tiennent des réunions de prières, de louanges et de témoignages; ils échangent sur des sujets bibliques ou des questions de foi personnelles en consultant librement des publications variées, qu'elles proviennent de la WTBTS ou d'autres éditeurs. Ces groupes sont animés par des «aînés » élus dans le but de présider les réunions et de distribuer la parole et à qui est, également, dévolue une fonction de prêche et d'enseignement auprès des autres étudiants.

Dans ces conditions, les classes sont assimilables à des « coopératives spirituelles ": en conservant leurs attaches confessionnelles antérieures et en pratiquant une lecture communautaire de textes soumis à commentaires, les étudiants de la Bible construisent un espace de discussion et de croyances dans lequel

15. Voir, par exemple, le cas d'Alexander Freytag (1870-1947) et de son mouvement « Les amis de l'homme ", qui est historiquement la branche suisse des étudiants de la Bible, dans Jean Vernette, Claire Moncelon, Dictionnaire des groupes religieux aujourd'hui, Paris, PUF, 2001, p. 8-9.

16. À partir de 1904, Russell lance un programme "d'évangélisation par la presse ». Pendant une dizaine d'années, il publie ainsi des sermons dans de nombreux journaux contre l'achat d'espaces publicitaires. En outre, à partir de 1914, les permanents de la WTBTS élaborent puis projettent de façon itinérante une animation cinématographique, le «photo-drame de la création ». Combinant un film et des vues fixes, et accompagnée d'enregistrements musicaux et de discours phonographiques, la projection retrace l'évolution de l'humanité, de la création jusqu'à la fin du millénium. À l'ère du cinéma muet, cette animation connaît un grand succès populaire. 
la plurivocité du corpus biblique est communément acceptée et l'adhésion aux conceptions de Russell non exclusive. À mesure que le mouvement se développe (si une trentaine de classes existent en 1880 , elles sont près de cinq cents en 1915, essentiellement aux États-Unis) et que l'année 1914 approche - et, avec elle, l'entrée espérée dans une nouvelle phase de l'histoire humaine -, la WTBTS insiste davantage sur le témoignage que les étudiants doivent donner auprès de la population. Cependant, en dépit des invitations réitérées de Charles Russell, très peu d'adhérents distribuent eux-mêmes les imprimés de l'organisation.

Afin de ramener les étudiants au message millénariste de la société de la Tour de Garde (à son examen et à son annonce), la WTBTS publie plusieurs imprimés en vue de structurer, à distance, les activités des groupes. Ainsi, en 1890, sort un ouvrage censé fournir une liturgie rudimentaire aux congrégations Poems and Hyms of Millenial Dawn (Poèmes et hymnes de l'aurore du millénium). De plus, à l'aide de questionnaires imprimés à la fin des volumes des Studies in the Scriptures, la WTBTS ambitionne d'organiser une réunion spécifique dans toutes les classes, pour acquérir des plans de discussion (questions et réponses) fondés sur la lecture scrupuleuse de chacun des volumes des Studies. Les pèlerins envoyés dans les classes ont, précisément, pour tâche de centrer les réunions sur la consultation ordonnée des Studies et des conceptions qui y sont promues et, conjointement, de réduire les capacités de prêche des aînés. Le projet religieux et éditorial de la WTBTS - tel qu’il se construit dans la durée - apparaît ici : non pas ériger les classes en lieux autonomes d'expertise et de discussion des prophéties bibliques, mais en faire, progressivement, des lieux d'étude dont l'activité cognitive est étalonnée par les publications de la WTBTS.

Toutefois, pendant la phase de gestation du mouvement de la Tour de Garde et la présidence de Charles Russell (des années 1880 à 1916), ce programme reste, largement, inabouti et les groupes de lecteurs ne s'ajustent qu'imparfaitement au dispositif éditorial et cultuel de la WTBTS. Avec les ressources qui sont les siennes (rédaction et publication des imprimés, travail et loyauté de ses placiers et pèlerins), la WTBTS tente d'organiser la carrière des imprimés en faisant en sorte que l'activité des groupes de lecteurs se fixe autour de l'usage régulier (au double sens d'habituel et de conforme à la pensée russellienne) des imprimés produits. Mais le maniement des imprimés n'organise pas la vie des classes, non seulement à cause des aléas de l'approvisionnement en imprimés WTBTS (dépendant des passages de colporteurs et des livraisons des imprimeries), mais aussi parce que chaque groupe local s'administre de façon indépendante.

Avec la mort de Charles Russell en 1916, et jusqu'au début des années 1940, le mouvement de la Tour de Garde entre dans une période de turbulences marquée par une crise de succession à la tête de la WTBTS, l'éclatement de la mouvance des étudiants de la Bible et la naissance (en 1931) d'une nouvelle formation, celle des Témoins de Jéhovah. Les travaux sociologiques et historiques sur le sujet appréhendent ces bouleversements comme une reconfiguration de 
l'organisation selon une logique sectaire, c'est-à-dire sa restructuration durable ${ }^{17}$ en un groupe socialement enclavé rassemblant des individus religieusement qualifiés. De nombreux traits attestent cette orientation, tant du point de vue de la doctrine professée par la WTBTS que des nouvelles pratiques de ses adhérents.

Le nouveau président de la maison d'édition, Joseph Rutherford (1869-1942) rompt avec l'attitude de son prédécesseur, peu intéressé par les événements du monde, et diffuse par l'intermédiaire de différents imprimés un corps de croyances de combat contre les institutions ecclésiales et politiques de son époque, accusées d'être animées par le Diable. Son virulent message d'insoumission aux pouvoirs publics est à la source de nombreux conflits dans le courant des années 1930, et de nombreux étudiants subissent alors la répression des autorités ${ }^{18}$. Entre outre, sous son mandat, la WTBTS et ses lecteurs sont investis d'une mission particulière : la proclamation incessante de la vérité et le combat spirituel contre les forces coalisées des ténèbres. Pour la première fois dans l'histoire de la pensée des étudiants de la Bible, l'organisation tient une place prépondérante dans le schéma eschatologique, celle d'un outil dans la main de Dieu pour informer l'humanité de son plan et participer à sa réalisation. La différence est de taille avec les enseignements de Russell, pour qui les activités de la maison d'édition et de ses lecteurs ne concourraient pas, historiquement, à l'accomplissement du «plan divin des âges ». Par ailleurs, la figure de Russell messager visionnaire est, progressivement, évacuée des publications de la maison d'édition, qui interrompt le commerce des photographies de Russell et arrête la circulation de ses écrits. Cette fonction de transcripteur des desseins divins est alors collectivisée à la WTBTS toute entière, qui se déclare en 1938 "théocratique ", c'est-à-dire seul instrument de communication de Dieu dans le monde.

Sur le plan des pratiques, les étudiants fidèles à la nouvelle direction de la WTBTS se coupent des autres chrétiens : la célébration de la fête de Noël est abandonnée en 1927, le symbole de la Croix, utilisé sous Russell, est délaissé dans le courant des années 1930 pour son paganisme supposé, et l'appartenance au mouvement implique, désormais, la rupture des liens religieux antérieurs. Ces transformations entraînent d'importants mouvements de défection : entre 1917 et 1921, environ la moitié des partisans de Russell quittent le mouvement des

17. Les principales caractéristiques de l'organisation se fixent pendant cette période. Se reporter à James BECKFORD, op. cit., chap. 2 et 3 et à Bernard BLANDRE, Les Témoins de Jéhovah, Bruxelles, éditions Brépols, 1991, p. 20 sq.

18. Ces tensions résultent du refus des fidèles de Rutherford de manifester publiquement leur subordination à l'ordre étatique (en accomplissant leur service militaire ou bien en effectuant le salut traditionnel au drapeau). Aux États-Unis, pendant la Seconde Guerre mondiale, deux mille étudiants sont incarcérés pour ces raisons. Voir James BECKFORD, op. cit., p. 35. Dans un autre contexte, mais pour des faits similaires, sous le régime nazi, plus de six mille partisans de Rutherford sont envoyés à partir de 1939 en camps de prisonniers ou en hôpitaux psychiatriques. 
étudiants de la Bible qui se fragmentent en une multitude de petits groupes. Des imprimés circulent largement, contestant la ligne doctrinale de Rutherford, et certains pèlerins en dissidence organisent des groupements d'étudiants séparés ${ }^{19}$. Ceux qui restent fidèles aux enseignements et aux consignes de Rutherford se spécifient, ainsi, en une formation aux croyances et aux pratiques distinctes et prennent le nom, en 1931, de Témoins de Jéhovah.

Cette entrée progressive dans une logique sectaire, brièvement esquissée, va de pair avec une refonte de l'univers productif et croyant organisé autour de la maison d'édition. S'extrayant de la matrice originelle des étudiants de la Bible, l'organisation des Témoins de Jéhovah se construit à partir d'un travail de régulation des conditions de production, de distribution et d'utilisation des imprimés. À la période russellienne, caractérisée par l'éclatement organisationnel et l'autonomie croyante des groupes d'étudiants, succède un moment de centralisation du mouvement, initié par le nouveau directeur Joseph Rutherford, visant à maîtriser l'ensemble de la carrière des imprimés (de leur fabrication à leur consultation, en passant par leur diffusion). Le projet, tel qu'il se réalise historiquement, est de créer une filière intégrée des imprimés, évitant les ruptures d'approvisionnement (à cause des problèmes d'articulation entre les univers de production et de distribution), et reposant sur le travail de colportage de chaque membre, dans tous les groupes locaux.

Le premier acte de cette entreprise de reconfiguration est la rupture des partenariats commerciaux précédents pour réaliser matériellement les imprimés. Dès 1919, le siège de la WTBTS, installé à New York dans le quartier de Brooklyn, s'équipe en machines, aménage des ateliers, et appelle des bénévoles à se consacrer à la confection des imprimés. Les fascicules puis les ouvrages sont, progressivement, produits intégralement par le siège de Brooklyn. À l'étranger, également, les groupes d'étudiants fidèles à la maison d'édition se dotent, sur recommandation du siège, de moyens d'impression. Ces opérations hors des États-Unis sont pilotées à distance par la WTBTS. Cette gestion mondiale de la production est assurée, de manière centralisée, par le bureau de Brooklyn qui décide de l'implantation des imprimeries, des quantités produites et des langues de traduction en fonction de la répartition géographique des lecteurs et des conditions politiques et économique régionales. Dans ce cadre, la WTBTS veille à conserver le monopole de la production d'imprimés, donc à empêcher chaque échelon (filiale nationale ou congrégation de base) de mettre en circulation ses propres imprimés. Tout aîné de congrégation organisant la publication d'une feuille indépendante (l'équivalent

19. Tels le Laymen's Home Missionary Movement (Mouvement missionnaire intérieur laïque) et le Pastoral Bible Institute (Institut pastoral biblique) principalement. Ces formations fonctionnent comme la WTBTS des origines mais à échelle plus réduite, c'est-à-dire organisés autour d'une maison d'édition qui approvisionne sur commande une nébuleuse de lecteurs à domicile ou en groupes. Voir Alan RogERSON, "Témoins de Jéhovah et Étudiants de la Bible. Qui est schismatique?", Social Compass, janvier 1977, vol. 24, nº 1. 
des bulletins paroissiaux catholiques par exemple) est sévèrement rappelé à l'ordre ou écarté de l'organisation.

En se lançant dans la production mondiale et coordonnée des imprimés, la WTBTS change de dimensions et de logique. Le système de production extensif qui se met, alors, en place (maillage mondial en équipements productifs, augmentation régulière des volumes d'imprimés) est soutenu par la maîtrise progressive du circuit de distribution et de ses dispositions à créer de nouveaux débouchés (par l'augmentation du nombre des lecteurs). Le passage à la production en propre est solidaire de la capacité de la WTBTS, construite difficilement dans les années 1920 et 1930, à faire reposer la diffusion des imprimés non plus sur un personnel spécialisé (colporteurs et pèlerins) mais sur tous les Témoins de Jéhovah pris individuellement, appelés à se faire les relais de la parole imprimée de Dieu auprès de leurs contemporains. Un enjeu adjacent est, également, de cadrer l'utilisation de ces imprimés chez les Témoins de Jéhovah, c'est-à-dire de rendre prévisible et répétitif l'usage des imprimés. Ces deux résultats sont obtenus, graduellement, grâce à la recomposition du circuit de distribution, qui est le deuxième versant du mouvement de centralisation.

Dès les débuts de la présidence de Rutherford, la WTBTS et ses agents mènent un travail socio-éditorial dont l'objectif explicite est de consolider la loyauté des groupes locaux (envers la WTBTS) et de structurer, à distance, leurs activités en les intégrant dans un programme du croire établi par le centre éditorial. D’un point de vue analytique, le sens de ces opérations est de subordonner les groupes de lecteurs à la WTBTS selon une double perspective : leur succursalisation dans le réseau de diffusion des imprimés, et la normalisation de l'utilisation des imprimés. En 1919, à l'occasion du lancement d'un nouveau magazine d'informations générales, Golden Age (L'âge d'or) ${ }^{20}$, la WTBTS pose des conditions drastiques aux classes d'étudiants (et non aux lecteurs individuels) pour en obtenir un approvisionnement régulier. Sur des bases contractuelles, le groupe local s'engage à diffuser activement le périodique auprès du grand public et à rendre compte régulièrement de son activité au bureau de Brooklyn. En outre, d'un point de vue logistique, les classes se transforment progressivement en centres de tri : tous les imprimés de la WTBTS, au lieu d'être portés à domicile par les colporteurs et les pèlerins (et parfois par la poste), sont centralisés dans les groupes qui les répartissent ensuite entre membres. De ce fait, la maison d'édition expédie des lots aux classes qui les répercutent en cascade auprès des abonnés. Ce système se met en place avec le lancement de Golden Age et permet à la WTBTS de programmer plus facilement sa production (en disposant de quantités provisionnelles), de garantir un écoulement régulier des imprimés et de faire l'économie d'intermédiaires. De surcroît, les imprimés dissidents (confectionnés

20. Le titre de ce périodique change en 1946 en Awake! (Réveillez-vous !). 
notamment par les étudiants de la Bible hors de la WTBTS ou par d'autres mouvements chrétiens) se raréfient dans les classes car ils sont exclus de ce système.

Afin de faire respecter les termes de cet accord, la société de la Tour de Garde établit une catégorie de bénévoles itinérants chargés de l'application locale des directives centrales. Ainsi, le service de colporteur et de pèlerin est remplacé en 1924 par le travail de surveillant. En outre, afin de réduire la marge d'action des aînés dans la lecture communautaire des imprimés, la WTBTS tente d'uniformiser les activités de lecture des classes en installant le périodique Watch Tower en point de passage obligé. En 1922, des plans de discussion qui visent à servir de cadres de l'interaction communautaire sont diffusés par l'intermédiaire du Watch Tower (sous la forme d'une rubrique régulière). De plus, les groupes sont incités à organiser des réunions hebdomadaires (réunion « d’étude de La Tour de Garde ») en calant leurs échanges sur le plan de discussion fourni par le périodique. Parallèlement, une épreuve s'engage entre le centre éditorial et les classes à propos de la désignation des aînés : un mécanisme de certification de ces derniers (un test écrit qui mesure leur orthodoxie) est mis en place et les groupes sont enjoints de choisir leurs responsables sur la base de leurs résultats à l'examen. Le point de rupture de cette dynamique de mise sous tutelle des classes est atteint en 1938 avec l'affirmation théocratique de la WTBTS. Sous peine de séparation définitive avec la maison d'édition, les groupes sont sommés de signer une déclaration stipulant l'abandon de leur droit de désigner électivement leurs aînés au profit du bureau de Brooklyn, seul en charge de les nommer à partir d'une liste d'aptitude.

En se pliant, progressivement, au dispositif social et éditorial impulsé par le bureau central de New York, les classes s'agrègent ainsi à un ordre des imprimés où celles-ci n'ont plus d'autonomie mais participent, selon des mécanismes réglés et telles des franchises, à l'activité de distribution et de déchiffrement des imprimés. Sous l'égide de la WTBTS et au prix de nombreuses défections, est mené un travail global de mise en convergence de deux ensembles auparavant disjoints et étrangers l'un à l'autre, la production et la diffusion des imprimés. Cette intégration, structurante historiquement pour le mouvement, est réalisée sur la base d'un impératif, inter-subjectivement partagé et régulièrement réitéré, de bonne circulation et de large dissémination des imprimés, porteurs d'une alarme particulière car apocalyptique.

\section{La figure de la centrale d'alarme}

Les réorganisations évoquées se réalisent sur fond d'alarme millénariste. Dès 1920, dans l'ouvrage Millions Now Living Will Never Die (Des millions de personnes actuellement vivantes ne mourront jamais), Joseph Rutherford se livre à une réinterprétation complète des prévisions russelliennes (notamment celles concernant l'année 1914), révise le mode de calcul en vigueur et proclame que 
«la fin visible du présent ordre de choses » est attendue pour l'année 1925. De même, au cours des années 1930, le nouveau président de la WTBTS annonce de manière incessante l'ouverture de la phase apocalyptique pour une période proche, mais cette fois sans avancer de date. Les formules d'avertissement, de maintien en éveil deviennent alors omniprésentes dans la littérature publiée par la société de la Tour de Garde. Après 1966, une nouvelle année est annoncée comme début de l'apocalypse : 1975.

Les recompositions organisationnelles, productives et croyantes des années 1920 à 1940 participent à un processus de mise en forme durable du mouvement en centrale d'alarme ${ }^{21}$. Cette expression désigne deux réalités solidaires. La première est que l'organisation émet, à flux réguliers, des messages de mises en garde qui oscillent entre les formes de l'alarme, de l'appel à la vigilance, et de la mise en éveil. La seconde est que ces messages sont transportés par une série d'intermédiaires (artefacts techniques, imprimés, Témoins de Jéhovah), strictement alignés les uns par rapport aux autres, de manière à ce qu’ils soient diffusés en évitant les modifications et les appropriations. Ainsi, les mises en garde imprimées transitent du centre éditorial vers les congrégations, et des membres assemblés vers le grand public, grâce à des formes et des relations qui limitent les possibilités de fluctuation et de reformulation individuelle.

L'intérêt initial des sociologues pour le mouvement jéhoviste a trait à ses alertes à la fin des temps, telles celles concernant les années 1925 et 1975 , et au fait que l'invalidation de ces prévisions ne s'est pas traduite concrètement par des départs massifs de fidèles. Empiriquement, ces alertes sont des messages qui, dans l'ensemble des publications, se présentent comme des résultats logiques de calculs chronologiques rénovés. Elles viennent corriger les scénarisations précédentes de l'histoire du salut et rétablir une datation du moment eschatologique déterminée dorénavant avec plus de justesse. Ces remaniements ne se donnent pas à évaluer comme de nouvelles révélations prophétiques, délivrées par des êtres choisis, mais comme les produits d'une connaissance cumulative des desseins divins. Un événement n'est pas à l'origine du lancement de l'alarme (une série de catastrophes, de calamités ou d'épidémies), seules une révision critique du mode de calcul, une correction circonstanciée de la " minuterie eschatologique ", justifient l'annonce du déclenchement historique de la phase apocalyptique. Ces alarmes sont centralisées. Bien qu'elles ne soient signées ou endossées par aucun acteur individuel ou collectif, elles sont émises par la voie des imprimés de la Tour de Garde, c'est-à-dire qu'elles sont, préalablement, accréditées par les plus hautes instances du mouvement. Bien que de nombreuses tâches d'impression soient confiées aux filiales nationales, tout le travail rédactionnel et éditorial

21. Pour saisir l'épaisseur sociologique des phénomènes d'alarme, voir Francis ChateauRaYNaud, Didier TORNY, Les sombres précurseurs. Une sociologie pragmatique de l'alerte et du risque, Paris, Éditions de l'EHESS, 1999. 
est centralisé à Brooklyn : les éditions étrangères ne sont, strictement, que les traductions de la version américaine, sans modification ni adaptation locale. Même les caractéristiques formelles (mise en page, illustrations ou découpage des textes) sont reproduites à l'identique. De la sorte, des formulations alternatives de l'alarme apocalyptique (ou des contestations de la pertinence de l'alerte lancée) ne peuvent circuler par la chaîne des imprimés de l'organisation, qui, en l'espèce, n'accordent aucune publicité aux propositions millénaristes concurrentes de celle de la WTBTS. Une alarme lancée unilatéralement par un Témoin de Jéhovah, voire par un groupe local, n'a aucune chance de cheminer à travers le mouvement parce que son auteur sera immédiatement exclu.

Si l'alerte est composée par un milieu de rédacteurs autorisés et relayée de manière descendante dans les groupes locaux par voie d'imprimés, elle reste cependant ambiguë sur le plan de sa plausibilité pratique. Lors de sa formulation dans un livret, elle n'est pas mise en relief sur le plan typographique, visuel ou narratif : par la suite, elle n'est que rarement reprise, répétée ou explicitée au sein de l'organisation. La WTBTS ne publie pas d'imprimés spéciaux pour son lancement, ni ne mène de campagne de presse particulière à mesure que l'année en question approche. De même, et en dépit de sa puissance d'évocation, de signification et de prémonition pour le lecteur, l'alerte ne vient nullement bouleverser le rythme ordinaire codifié des activités et des réunions des Témoins de Jéhovah, même si elle peut donner lieu à commentaires et à échanges informels. Ce paradoxe d'une alerte radicale lancée mais, pratiquement, non considérée comme telle, éludée, sur laquelle l'attention n'est pas ramenée, est à mettre en perspective avec les caractéristiques de l'idiome millénariste, dans lequel la durée est vécue comme un état de présence à un intervalle de temps téléologique, finalisé $^{22}$. Les acteurs traversent une durée qui se raccourcit, qui tend à son épuisement. L'expérience de la durée est orientée vers le surgissement d'un événement attendu et inexorable, l'ouverture du moment apocalyptique. Dans ce cadre, la société de la Tour de Garde ne cesse d'aviser ses membres que le couplage des temps historique et eschatologique est arrivé, même si, par intermittence, elle le signifie plus nettement en avançant une date. Aussi, lorsque l'année indiquée est dépassée (comme ce fut le cas pour 1925 et 1975), l'organisation n'opère pas de manière claire de retour critique, ni ne réestime la pertinence de l'alerte. Celle-ci est plutôt traitée comme participant du flux des mises en garde et des avertissements réguliers adressés aux Témoins de Jéhovah pour leur rappeler l'horizon temporel dans lequel leur action se situe. De cette manière, même si des butoirs temporels précis sont parfois avancés, puis traités ultérieurement par le silence, une dynamique de l'urgence, de l'imminence de l'Apocalypse est constamment poursuivie.

22. Consulter Raymond Duval, Temps et vigilance, Paris, Vrin, 1990. L'auteur présente la gamme des formes de présence à la durée : le délai, l'urgence, le compte à rebours, l'écoulement monotone de l'attente, la durée anonyme du temps qui ne passe plus. 
L'homologie entre le mouvement de la Tour de Garde et la centrale d'alarme mérite d'être considérée, à titre heuristique, pour une seconde raison, qui tient aux modes de transmission des messages d'alerte et d'urgence. L'organisation interne des Témoins de Jéhovah, ainsi que les formes de prédication qu'ils utilisent à l'égard du grand public, sont aménagées de telle manière que le message de la WTBTS circule sans altération, sans variation. À la suite des restructurations des années 1920-1940, la société de la Tour de Garde se spécifie en une filière intégrée des imprimés qui relie hiérarchiquement un centre (fabriquant, matériellement et sémantiquement, des alertes imprimées) à une multitude de congrégations franchisées et alignées par rapport au dispositif socio-éditorial de la WTBTS. Dans ce cadre, les fidèles ordinaires n'ont pas vocation à participer à l'élaboration des messages, par exemple en faisant remonter vers le centre éditorial des commentaires ou des formulations millénaristes alternatives. Ils interviennent, plutôt, en tant que surveillants du dispositif de transmission des alertes (vérifier le travail de chacun, faire respecter les procédures de distribution et de lecture des imprimés), et en tant qu'opérateurs chargés de la maintenance de la chaîne de diffusion (production et approvisionnement en publications, organisation des réunions et des séquences de prédication). En outre, pour que les messages d'éveil et d'urgence soient transportés sans détérioration, les médiateurs sont alignés les uns par rapport aux autres. Ad intra, un circuit de distribution des publications se forme, évacuant toute littérature extérieure à la WTBTS. Le traitement (l'attention, le mode de lecture) donné aux imprimés tend à la normalisation : le travail social et éditorial de la WTBTS contribue à faire converger les Témoins de Jéhovah vers des formes de réunions et de lectures - d'échanges sociaux contenus dans les périodiques sous les traits de plans de discussion à suivre et contrôlés par des surveillants. Ad extra, des dispositifs techniques diversifiés sont actionnés par des Témoins de Jéhovah, tenus par des protocoles d'interaction comprimant la dimension personnelle de la prédication.

Ainsi, jusqu'aux années 1950, la société de la Tour de Garde limite les possibilités d'énonciation individuelle de son message en utilisant des artefacts techniques pour sa diffusion vers le grand public. Des stations radio sont mises en place et retransmettent des programmes préenregistrés et des conférences de Rutherford $^{23}$. La prédication se fait, alors, par l'intermédiaire du poste de radio, dans chaque foyer branché, en ne mobilisant pas significativement les Témoins de Jéhovah. Mais la contrepartie est qu'il est impossible de retenir de façon performante les auditeurs aux émissions. Si la parole de l'organisation est transmise sans altération chez les particuliers, elle ne peut se fixer efficacement dans leur esprit : aucune trace ne demeure des émissions et du message passé : le degré d'attention et le mode d'écoute des programmes sont largement indéterminés. En complément, la société de la Tour de Garde se lance dans la production de phonographes portatifs et pousse ses membres à se munir de cet équipement

23. La première est la WBBR, installée à New York, qui fonctionne de 1924 à 1957. 
pour répandre son message : en 1940, plus de quarante mille machines sont en service. Cette technique est conçue afin de mettre tous les Témoins de Jéhovah en mouvement de prêche, sillonnant les localités et exposant le message du mouvement dans chaque maison, et ceci avec un savoir-faire très limité dans l'art de la prédication. En se présentant au seuil d'une habitation, le Témoin de Jéhovah demande simplement si ses interlocuteurs sont disposés à écouter une communication importante, puis installe son matériel, choisit un disque (de brefs discours enregistrés et fournis par la maison d'édition), et pose l'aiguille sur celui-ci. Pendant l'audition de l'allocution, le prédicateur attend sans exprimer aucune émotion : il administre un message; si les auditeurs manifestent de l'intérêt au message, ils sont invités, par la suite, à une réunion publique rassemblant des novices. Aussi, pendant les actions de prédication, les Témoins de Jéhovah n'articulent eux-mêmes aucun message d'alerte et d'urgence du mouvement : aucun de leurs gestes ou de leurs paroles ne signifient que les derniers temps sont proches et peuvent intervenir à tout instant. Les membres de la WTBTS portent à la connaissance du grand public une alerte formatée, exprimée dans le code utilisé par l'organisation : ils sont les vecteurs impassibles d'un avertissement transcodé.

Cette même logique est à l'œuvre dans la distribution porte-à-porte d'imprimés, technique de prédication qui se développe lentement à partir des années 1940 et se substitue progressivement aux précédentes. Le portage d'imprimés (le plus souvent des brochures et magazines jéhovistes) est l'objet d'une préparation minutieuse au sein des congrégations : les territoires de prospection sont périodiquement actualisés et répertoriés, et les Témoins de Jéhovah apprennent par cœur (à partir de livres et de feuilles d'instructions) des répliques standards, des entrées en matière type afin de structurer l'interaction d'évangélisation. Le démarchage à domicile, toujours effectué par deux, n'a pas pour but d'écouler de façon indiscriminée un lot d'imprimés, mais de les placer dans des foyers susceptibles de les lire et de recevoir, ultérieurement, les prédicateurs. Lors de la rencontre de colportage, l'appareil de persuasion gestuel et langagier du prédicateur n'est pas orienté vers la construction d'une relation interpersonnelle, qui pourrait déborder le cadre du moment d'évangélisation, mais vise à amener la personne visitée vers l'imprimé offert : le Témoin de Jéhovah manipule ses imprimés sous les yeux du profane, pratique des lectures oralisées devant lui, et tente de le faire participer aux plans de discussion figurant dans les publications. Il revient ensuite chez les personnes bien disposées pour leur proposer une série d'études bibliques à domicile, en s'appuyant sur des fascicules prévus à cet effet. Ainsi, l'interaction d'évangélisation est balisée par les imprimés : le prédicateur met en œuvre des recommandations, s'intègre à des plans d'interaction contenus dans les imprimés, sans que sa «subjectivité » (la manière dont il vit et entend ce message) ne soit mise à contribution dans la présentation du message jéhoviste. En termes théologiques, les Témoins de Jéhovah n'ont pas à être personnellement des signes, de 
la promesse de Dieu auprès du grand public, mais à transporter, sans distorsion, une information d'avertissement concernant la proximité d'un grand dénouement. La transmission ne varietur d'un message, grâce à l'alignement d'un réseau de médiateurs (artefacts techniques jusqu'aux années 1950, imprimés, Témoins de Jéhovah), est ainsi la raison d'être de l'action de prédication ${ }^{24}$.

Si le mouvement de la Tour de Garde s'organise, à partir des années 19201940 , en une centrale d'alarme et d'avertissement dans laquelle les messages ont vocation à circuler de proche en proche sans reformulation, les conditions concrètes de réception de ces messages par les Témoins de Jéhovah restent à élucider. De quelle manière les possibilités individuelles d'idiosyncrasie et de conduites non recommandées sont elles restreintes? Cette question renvoie aux modalités de constitution de l'expérience jéhoviste, aux formes dans lesquelles les croyances sont vécues et énoncées. En l'espèce, elle invite à comprendre comment les imprimés - compte tenu de leur statut et des pratiques sociales qui s'en emparent - construisent un mode de croire particulier qui est mémorisation et répétition des messages de la WTBTS.

\section{La structuration de l'expérience jéhoviste par l'imprimé}

L’investissement massif des Témoins de Jéhovah dans le secteur éditorial contraste avec le faible intérêt porté à d'autres techniques de prédication plus innovantes. Ainsi, la WTBTS ne produit que depuis peu, et dans des quantités limitées, des cassettes audio et vidéo et des CD-ROM. Peu variés, uniquement réservés aux membres assidus (non proposés lors du porte-à-porte), leur écoute et leur visionnage n'exemptent pas les membres de lire les publications ni d'assister aux réunions de leur congrégation de rattachement. Dans l'approche du grand public, l'usage du téléphone n'est autorisé qu'occasionnellement, en cas d'impossibilité pour le colporteur d'avoir accès au domicile visé ${ }^{25}$. Le prédicateur n'a pas vocation à démarcher, systématiquement, par ce médium, ni à inciter son interlocuteur - dans l'interaction téléphonique, sous forme d'allocutions préenregistrées ou d'échanges verbaux entre l'appelant et l'appelé - à croire à l'imminence du moment apocalyptique et à passer des commandes de publications. Le portage de la littérature jéhoviste à domicile, le cycle d'études bibliques entre l'évangélisateur et le profane, et le raccordement à la congrégation demeurent des séquences d'action dûment recherchées par la WTBTS. Des restrictions d'utilisation pèsent tout autant sur internet, espace de communication dont la

24. Cette configuration est à comparer avec d'autres régimes de transmission en monde croyant, dont la logique n'est pas de propager sans déformation un signifié originel mais de faire participer un destinataire au surgissement hic et nunc de la parole de Dieu et à créer de nouvelles formes (de cette présence divine) par appropriations successives. Consulter Bruno LaTOUR, "Quand les anges deviennent de bien mauvais messagers ", Terrain, mars 1990, no 14.

25. Lorsque la personne sollicitée est malade, ou lorsqu'un dispositif de sécurité empêche l'entrée d'un immeuble. 
fréquentation à des fins d'évangélisation est déconseillée aux Témoins de Jéhovah ${ }^{26}$. L'échange d'études bibliques au sein de forums de discussion est vivement découragé au motif que l'identification des participants y est aléatoire, et qu'ainsi des Témoins de Jéhovah peuvent - insensiblement et dans un climat de confiance mettre leurs croyances en débat avec un intrus, les hybrider ou les corroder hors du contrôle de la congrégation de base. Cette faible diversification des supports du message jéhoviste, les limitations d'utilisation qui affectent ces moyens de communication récents, trouvent leur pendant dans l'affirmation récurrente de la primauté des congrégations locales en tant que lieux dans lesquels s'effectue une lecture ordonnée des imprimés de la WTBTS, se prépare la distribution de porte-à-porte, et se consolident les croyances jéhovistes.

À côté des quatre heures hebdomadaires de porte-à-porte, l'appartenance à l'organisation s'exprime à travers l'assistance assidue aux réunions de la congrégation, d'une durée cumulée de cinq heures par semaine pour cinq réunions. Ces réunions sont beaucoup plus suivies que dans d'autres groupes religieux : comme chacun se connaît, un absentéisme régulier mène, progressivement, à l'éviction. Ces rassemblements, se tenant le plus souvent dans des locaux appelés «salles du Royaume ", suivent un déroulement identique dans tous les pays où le mouvement est implanté. Les imprimés sont au centre de la conduite des membres. Deux réunions sont, spécifiquement, consacrées à la préparation du colportage : des feuilles d'instruction sont lues à cet effet, des entrées en matière préconisées sont répétées, et les lots d'imprimés sont répartis entre les fidèles (qui disposent d'un compte personnel et d'un compte pour leur travail d'évangélisation). Deux autres rassemblements hebdomadaires sont littéralement adossés aux publications dans la mesure où les activités de lecture et de parole des participants, et l'ordre de leurs échanges, sont inscrits dans les publications, qui balisent alors l'interaction communautaire.

Il en est ainsi de L'enseignement à l'étude de La Tour de Garde, le magazine du mouvement auquel tout Témoin de Jéhovah est nécessairement abonné. À chaque réunion hebdomadaire correspond, en vis-à-vis, un article précis contenu dans le périodique. Le mode de découpage du texte indique le mode de lecture et le type d'échange auxquels la consultation de l'article donne lieu. Le texte à consulter est divisé en séquences brèves et closes sur elles-mêmes. Chaque partie de texte, très courte, renvoie, en bas de la page, à une ou deux questions qui portent sur des passages du paragraphe visé et auxquelles on demande des réponses très proches du texte, le plus souvent des paraphrases ou des reprises du texte.

26. Se reporter particulièrement au supplément de ministère du Royaume, novembre 1999 (feuille de conseils de prédication envoyés aux seuls Témoins de Jéhovah). Le site internet du mouvement (www.watchtower.org), bien qu'il affiche des articles de périodiques et des extraits de livres, n'organise aucune sociabilité particulière (pour les Témoins de Jéhovah ou pour le grand public), mais il oriente, en dernier recours, le visiteur vers la branche nationale du groupe, qui l'aiguille ensuite vers la congrégation relevant de son lieu de résidence. 
À la fin de l'article, des questions de révision dans un encadré achèvent la lecture. Un conducteur de l'étude, le plus souvent responsable de la congrégation, a pour mission d'assurer le bon déroulement de la réunion en ramenant ses coreligionnaires au texte lu et en se tenant strictement à l'ordre décrit par le périodique. L'imprimé constitue la trame du rassemblement en scandant son avancement, marqué par l'enchaînement des paragraphes, ponctué par l'alternance de questions et de réponses. Après la lecture de chaque partie, un fidèle demande à répondre à la question et, s’il est interrogé, répète, paraphrase ou résume le morceau de texte déchiffré : le conducteur corrige les erreurs éventuelles et repose la question tant qu'il n'a pas obtenu la bonne réponse. Puis l'assemblée vérifie dans l'article la conformité de la réponse et la lecture poursuit son cours. Pendant cette étude, le conducteur joue le rôle d'opérateur du mécanisme induit par l'architecture de l'article imprimé : il ne doit pas interpréter le texte ou le donner à apprécier par une médiation subjective, mais être le garant de la conformité littérale des réponses obtenues. Une telle physionomie de l'activité de lecture communautaire se retrouve pendant "l'enseignement à l'étude du livre ". Chaque année, l'organisation détermine l'ouvrage examiné, et les passages à consulter en groupe sont mentionnés chaque mois dans un feuillet spécial. Ici aussi, la lecture du livre est linéaire mais hachée et donne lieu, sous la responsabilité d'un conducteur, à une alternance de questions et de réponses très proches du texte.

Une dernière réunion, enfin, «l'enseignement à la réunion publique », consiste en un discours d'un orateur expérimenté choisi à l'avance : pour traiter du thème donné, le conférencier s'appuie abondamment sur des références issues des publications jéhovistes et émaille son argument de versets bibliques mentionnés dans la littérature de la WTBTS. Un des objectifs de ce rassemblement est de mesurer les capacités d'exposition de certains membres, pour leur attribuer des responsabilités plus importantes dans la congrégation, et s'assurer de leur orthodoxie (leur utilisation des imprimés, leur mode d'appropriation des textes de la WTBTS). Dans cet optique, le bon orateur est celui qui construit son allocution à partir des textes publiés par le mouvement, en empruntant et assemblant des passages sans reformulation personnelle ${ }^{27}$. Sur cette base, il est possible de caractériser le mode de lecture que les Témoins de Jéhovah déploient à l'égard des imprimés qu'ils consultent : ceux-ci (essentiellement le magazine La Tour de Garde, les brochures et les ouvrages conseillés) font l'objet de lectures multiples, individuellement en silence à domicile (notamment pour préparer une réunion) et à voix haute en congrégation (ou en famille). La pratique de lecture des Témoins de Jéhovah présente la singularité de rendre inopérante la distinction classique entre

27. Pour une présentation plus détaillée de ces réunions, se reporter à Régis DERICQUEBOURG, "Le jéhovisme: une conception comportementaliste de la vie religieuse ", Archives de sciences sociales des religions, juillet-septembre 1986, $\mathrm{n}^{\circ}$ 62. Et Andrew HoLDEN, Jehovah's Witnesses. Portrait of a Contemporary Religious Movement, Londres-New York, Routledge, 2002, p. 64 sq. 
deux modèles de lecture opposés ${ }^{28}$, la lecture "intensive ", appuyée sur l'écoute et la mémoire, révérencielle et respectueuse, typique des situations où les livres sont rares, et la lecture "extensive ", qui consomme beaucoup de textes, passe avec désinvolture de l'un à l'autre, donne une moindre sacralité à la chose lue. En effet, les membres du mouvement sont censés lire une multitude d'imprimés en une année. Outre les vingt-quatre numéros annuels de La Tour de Garde, ils consultent l'ouvrage retenu pour la réunion d'étude de livre et doivent posséder les livres examinés pour "l'école du ministère théocratique " (une des deux réunions de préparation de la prédication ${ }^{29}$. D'une année à l'autre, de nouveaux imprimés paraissent et les Témoins de Jéhovah doivent en disposer.

La façon de lire en congrégation, conjuguée aux formes données aux textes, montre que l'objectif de la lecture jéhoviste est la mémorisation, l'absorption de textes en vue de leur restitution : lorsque l'on voit un livre déjà utilisé par un Témoin de Jéhovah, il est fréquent de trouver de longs passages soulignés dans la plupart des pages. La lecture est captation et rétention d'informations, pour les produire ultérieurement à un tiers. Ceci s'explique par le fait que les Témoins de Jéhovah sont indissociablement des fidèles mais aussi, selon les termes mêmes de l'organisation, des «ministres ordonnés » aptes à conduire des études bibliques aux domiciles des personnes intéressées à l'issue d'une visite porte-àporte. Ainsi, les Témoins de Jéhovah ne forment pas uniquement une communauté de lecteurs, unis par le partage d'un même mode de lecture sur un même corps d'imprimés, mais également une communauté de répétiteurs, capables de rendre intégralement un texte appris à une personne extérieure. S'ils adoptent un tel mode de lecture des imprimés, qui sont pour eux partis prenants d'une œuvre d'instruction mondiale, c'est que ces mêmes imprimés constituent, de surcroit, le principal dispositif de validation de leurs croyances.

Il n'est pas incongru de parler ici, pour le mouvement de la Tour de Garde, d'un dispositif éditorial du croire. L'utilisation presque exclusive des objets imprimés pour porter le message jéhoviste, l'approvisionnement régulier en imprimés par la voie des congrégations (dans lesquelles s'exerce un mode de lecture axé sur la mémorisation), convergent pour désigner les imprimés comme des objets qui mettent en forme l'expérience jéhoviste. Le statut qui leur est attribué, ainsi que leur rédaction, confirment cette analyse. Tandis que l'organisation appelle à s'abstenir de toute implication personnelle dans le cours du monde (engagements politiques, associatifs, caritatifs), la lecture des imprimés de la WTBTS, individuellement et en groupe, est, en effet, avec la prédication, une des observances majeures au sein des Témoins de Jéhovah. En outre, aucun élément du monde matériel n'est investi sur le plan religieux, c'est-à-dire organisé et vécu comme médiateur avec un ordre transcendant. L'ambiance des réunions

28. Roger CHARTIER, Culture écrite et société, op. cit., p. 146 sq.

29. Pour l'année 1991 par exemple, cinq ouvrages étaient au programme de cette réunion. 
est studieuse et dénuée de mysticisme ou de manifestations spectaculaires. La liturgie des réunions est très dépouillée, l'architecture des «salles du Royaume » (tant intérieure qu'extérieure) est dépourvue de tout signe religieux, les objets et images de dévotion n'ont pas cours. En revanche, les publications sont érigées en transcripteurs fidèles du plan historique de Dieu, et se présentent à la lecture comme des instruments qui démontrent la véracité des messages de l'organisation. En prêtant attention, ici, aux conditions d'écriture des textes, il apparaît que la visée des rédacteurs est de leur conférer, comme à des textes scientifiques ${ }^{30}$, un niveau élevé d'objectivité, de factualité, par certaines particularités narratives et formelles, la première étant l'anonymat de tous les textes jéhovistes. Cette règle de l'anonymat, en vigueur depuis 1942, empêche le lecteur de comprendre un texte, ou un corpus, en référence au travail d'un auteur et à son identité biographique. De ce fait, les textes anonymes empêchent toute dérivation vers la personne des auteurs ou la découverte d'une œuvre (l'ensemble de textes attribuables à un auteur identifié) ${ }^{31}$. Ainsi, sur les couvertures ou les premières pages des publications jéhovistes, ne figurent que le titre de l'imprimé et la maison d'édition, présentée non comme l'auteur mais comme l'organisme chargé de la publication des textes, tel l'instrument d'une vérité universelle ${ }^{32}$.

Ce mode d'exposition des textes jéhovistes est conforté, dans un second temps, par un type d'écriture qui, par l'absence de pronoms personnels et d'adverbes de temps et de lieu, dépouille les énoncés de toute référence à un rédacteur déterminé. Le travail d'écriture, en lui-même, est tenu au second plan par la sobriété du vocabulaire et du style. Composés, sur le plan typographique, avec de nombreux repères visibles (exergues, résumés récapitulatifs, illustrations en vignette...), les textes se veulent démonstration simple, et fondée empiriquement, du couplage des temps historique et apocalyptique. Aussi, ces textes (surtout ceux destinés aux profanes lors du porte-à-porte) sont-ils, typiquement, construits autour d'une succession de faits et commentaires (issus de livres et de journaux) et de citations bibliques appropriées. Avec un tel parti de narration, alternant sources savantes choisies, manipulées selon les conventions d'usage (respect des règles de citation, distanciation énonciative par rapport à la source) et renvois

30. Pour une analyse des pratiques d'écriture qui participent à la production des faits et des énoncés scientifiques, voir Dominique VINCK, Sociologie des sciences, Paris, Armand Colin, 1995 , p. 16 sq.

31. Sur la notion d'auteur, et sur sa fonction classificatoire sur les discours, voir "Qu'est-ce qu'un auteur ? ", in Michel Foucault, Dits et écrits 1954-1988, tome 1, Paris, Gallimard, 1994.

32. L'avis au lecteur du périodique La Tour de Garde affirme: "Le but de La Tour de Garde est d'exalter Jéhovah Dieu, le Souverain Seigneur de l'univers. Ce périodique suit la marche des événements mondiaux qui réalisent les prophéties de la Bible. Il s'attache à consoler tous les peuples par cette bonne nouvelle : le Royaume de Dieu fera bientôt disparaître ceux qui oppriment leur prochain et transformera ensuite la terre en paradis. En outre, il encourage ses lecteurs à croire en Jésus-Christ - le Roi régnant établi par Dieu -, celui qui, en versant son sang, a ouvert à l'humanité le chemin de la vie éternelle. Ce périodique, publié par les Témoins de Jéhovah, paraît depuis 1879. Il est politiquement neutre. Pour lui, seule la Bible fait autorité. » 
scripturaires, le lecteur suit un raisonnement qui ne se donne pas à voir comme une trajectoire de pensée individuelle, mais comme un argument impersonnel doté d'une double légitimité factuelle et biblique. À titre d'illustration, un article de La Tour de Garde avance :

"Croyez-vous que nous sommes dans les "derniers jours" ? John Garraty et Peter Gay (dans The Columbia History of the World) posent cette question : "Assistonsnous à la chute de notre civilisation ?" Puis ces historiens analysent les difficultés du pouvoir politique, l'augmentation, à l'échelle planétaire, de la criminalité et de la résistance passive, l'effondrement de la famille, l'échec de la science et de la technologie face aux problèmes de société, la crise de l'autorité, la dégradation morale et religieuse dans le monde entier. Et de conclure: "Si ce ne sont pas là les signes d'une fin claire et radicale, en tout cas cela y ressemble étrangement". Nous avons des raisons valables de croire qu'une “fin” est imminente. Nul besoin, cependant, de craindre la fin de la planète, car la Bible dit que Dieu "a fondé la terre sur ses lieux fixes ; elle ne chancellera pas pour des temps indéfinis, oui pour toujours". (Psaume 104, 5) En revanche, nous devons nous attendre à la fin prochaine du système de choses méchant qui depuis longtemps apporte tant de malheurs aux humains. ${ }^{33}$

Dans les publications consacrées aux membres (la plupart des ouvrages et certains articles de La Tour de Garde), les références à des travaux de recherche profanes, venant garantir un fondement factuel au croire jéhoviste s'estompent au profit d'une profusion de renvois bibliques. Ici, le mode d'utilisation des références bibliques mérite l'attention. En effet, dans le dispositif qui donne à voir le texte, il n'y a pas de séparation visuelle entre les passages empruntés à l'Écriture et les portions de textes attribuables à la WTBTS. Il n'est pas possible de faire la différence entre les positions propres de l'organisation, dûment assumées comme telles, et les emprunts scripturaires. Il n'existe aucun marquage typographique discriminant des versets bibliques mobilisés (par la mise en page, l'utilisation de caractères différents, etc.). De plus, le style des lignes imputables à l'organisation ne diffère pas de la forme biblique, mais s'apparente souvent à la paraphrase et la répétition. Dans les textes jéhovistes à aucun moment n'est visible le travail de l'auteur, qui choisit des passages bibliques, effectue des rapprochements entre eux, explique en détails son emploi des Écritures. Tout commentaire personnel sur les propriétés intrinsèques des Écritures, sur la façon de les comprendre et de les utiliser pour expliciter une croyance est exclu du champ de la narration. Les textes se donnent à lire comme des paraphrases des Écritures, déclinées et déroulées sous les yeux du lecteur par l'abondance de références bibliques. Dans un autre article de La Tour de Garde, il est écrit :

«La crainte de Dieu n'a rien d'une peur morbide. C'est plutôt un respect profond et une crainte mêlée d'admiration. Il ne peut y avoir de vraie connaissance sans cette crainte. La vie vient de Jéhovah Dieu, et il faut évidemment être vivant pour posséder une connaissance (Psaume 36, 9 ; Actes 17, 25, 28). En outre, Dieu a créé toutes choses: toutes les connaissances humaines ont donc pour point de départ une étude

33. La Tour de Garde, 15 septembre 1998, édition française, p. 5. 
de son œuvre (Psaume 19, 1, 2 ; Révélation 4, 11). Dieu a également inspiré sa Parole écrite, qui est "utile pour enseigner, pour reprendre, pour remettre les choses en ordre, pour discipliner dans la justice" (2 Timothée $3,16,17) .{ }^{34}$

Ainsi, les notes bibliques ne figurent pas au bas des pages, accompagnées de commentaires appropriés, mais sont incrustées dans le corps du texte pour lui conférer sa solidité. Leur place en fin des phrases, sous forme de référence, consacre la narration comme méthode d'argumentation déductive : à chaque assertion correspond, en vis-à-vis, une inscription scripturaire qui la fonde. De cette manière, par rapport au lecteur, les renvois bibliques ne fonctionnent pas simplement comme des renforts à la démonstration, mais délimitent le lieu d'où la pertinence des textes doit être évaluée; ils désignent la source de toute légitimité et de toute confiance en matière de sûreté des connaissances, les Écritures bibliques. Par ailleurs, ils déterminent la méthode adéquate de vérification de la véridicité des énoncés et leur ressemblance avec la lettre biblique. Ce travail, typographique et narratif, de mise en concordance du texte jéhoviste avec les textes bibliques investit ce texte d'un surcroît d'autorité dans la mesure où il s’institue comme intrinsèquement solidaire de la Bible, traversé de part en part de versets bibliques restitués sans commentaire sur le mode de la paraphrase. Ce dispositif éditorial qui présente les textes comme épousant avec exactitude la forme des énoncés bibliques - sans interstice, sans espace propre d'interprétation ou de commentaire - est le garant de la pertinence du croire jéhoviste.

En effet, pour les Témoins de Jéhovah, l'organisation à laquelle ils adhérent ne porte pas, ne formule pas de croyances particulières mais promeut de manière impartiale des vérités écrites directement assignables à la Bible. Les énoncés relatifs, par exemple, au rôle de la WTBTS et de ses membres dans l'histoire du salut, ou au déclenchement imminent et daté historiquement de la phase apocalyptique, ne sont acceptés et proférés que dans la mesure où la WTBTS les rapporte immédiatement à des versets bibliques précis. De ce fait, les croyances jéhovistes sont énoncées non pas sur un mode métaphorique (par emprunt ou analogie partielle avec des énoncés bibliques, considérés comme constituant un récit symbolique), mais sur l'affirmation de leur plein établissement biblique par certification scripturaire. Cette confusion des sources bibliques et jéhovistes franchit une étape décisive en 1961 avec la parution, puis la diffusion sur une large échelle, d'une traduction indigène de la Bible, New World Translation of the Holy Scriptures (Saintes Écritures-Traduction du monde nouveau). Plusieurs options doctrinales jéhovistes sont incorporées directement dans la traduction biblique, et certaines croyances qui différencient le mouvement de la Tour de Garde des autres Églises chrétiennes (telles notamment la mortalité de l'âme, la nature non trinitaire de Dieu) trouvent inscription dans cette traduction par des remaniements de ponctuation ou des formulations alternatives ${ }^{35}$. La Traduction $d u$ monde nouveau

34. La Tour de Garde, 15 septembre 1999, édition française, p. 13-14.

35. Sur cet aspect, se reporter à Bernard BLANDRE, op. cit., p. 3 sq. 
circonscrit alors l'espace des interprétations et des traductions bibliques acceptables au sein du mouvement dans la mesure où elle sert d'étalon : toutes les références bibliques mobilisées dans les publications jéhovistes sont directement issues de cette traduction qui, en outre, remplace progressivement toutes les autres traductions de la Bible (notamment celles qui sont acceptées par les catholiques ou les protestants) auparavant en circulation chez les Témoins de Jéhovah. En intégrant, ainsi, dans la lettre biblique ses partis théologiques, la WTBTS se dote d'un ouvrage de référence central sur lequel tous les autres imprimés s'appuient pour démontrer aux lecteurs l'ancrage biblique de leurs énoncés et, solidairement, la facticité des propositions croyantes concurrentes. L'anonymat des auteurs ainsi que le dispositif visuel et narratif qui présente les textes jéhovistes comme inséparables de la lettre biblique consacrent ainsi un mode de croire fondé sur la lecture et la mémorisation des imprimés.

Au terme de cette exploration, il apparaît que les objets imprimés tiennent un rôle spécifique tant dans l'histoire que dans le fonctionnement présent des Témoins de Jéhovah. Ils sont, ainsi, les intermédiaires par lesquels le mouvement de la Tour de Garde se transforme à partir des années 1920, quittant la forme d'un réseau de lecteurs pour adopter celle d'une centrale du croire, sur la base du souci de formaliser les conditions de production et de circulation des imprimés, vecteurs d'une alerte eschatologique. Ces mêmes imprimés forment aujourd'hui l'armature du lien social au sein de l'organisation jéhoviste, compte tenu des observances qui régulent leur acheminement, leur lecture et leur distribution. Leur consultation en groupe et leur diffusion auprès du grand public organisent une sociabilité et un mode de croire particuliers. En renouvelant l'approche du mouvement des Témoins de Jéhovah, cette entrée par les imprimés est susceptible de proposer de nouvelles perspectives d'analyse aux sciences sociales du religieux. Tout d'abord, elle autorise aisément les comparaisons avec d'autres ensembles religieux fondés sur la circulation d'imprimés, comme les sociétés d'édition biblique : la variété des pratiques en matière de rédaction, de diffusion et d'utilisation des différents imprimés pourrait alors mettre en relief la diversité des actions qui se jouent en situation autour des objets imprimés. Enfin, en prolongement, l'attention accordée aux dimensions matérielles de l'activité religieuse ${ }^{36}$ invite à prendre en considération la pluralité des objets et des dispositifs techniques qui construisent la diversité des expériences et des groupes croyants. En examinant le travail de régulation de leurs carrières et de leurs usages, l'enquête pourrait ainsi apporter un éclairage complémentaire sur les mécanismes d'affirmation et de validation des croyances.

Arnaud BLANCHARD

GAPP - CNRS/ENS Cachan

36. La démarche s'inspire ici de Jean-Pierre WARNIER, Construire la culture matérielle, Paris, PUF, 1999. 


\section{Résumé}

Afin de proposer de nouvelles pistes de recherche sur l'organisation des Témoins de Jéhovah, celle-ci est étudiée à partir des imprimés qu'elle produit et diffuse massivement. La participation au mouvement de la Tour de Garde correspond, en effet, à un ensemble de pratiques sociales liées aux objets imprimés (fabrication en grandes quantités, distribution de porte-à-porte, lectures individuelles et en groupe). Aussi, l'examen des relations sociales qui s'organisent autour des imprimés apporte un éclairage nouveau à la fois sur la trajectoire historique de l'organisation et sur son fonctionnement présent. Avec cette approche, les imprimés apparaissent comme des intermédiaires qui mettent en forme et structurent l'expérience jéhoviste.

Mots clés: Témoins de Jehovah, presse religieuse, prosélytisme, organisation religieuse.

\section{Abstract}

To propose a new approach to study Jehovah's Witnesses, their organization is scrutinized through the materials it prints and disseminates widely. Participation in the Watch Tower movement corresponds with a set of social practices linked to printed objects (production in high amounts, door-to-door canvassing, individual and collective readings). Thus, the examination of social relations organized around printed materials brings a new highlight both on the historical trajectory of the organization and on the way it currently works. Under this approach, printed materials appear as intermediaries which shape and structure the Jehovist experience.

Key words: Jehovah Witnesses, religious press, religious organization, proselytism.

\section{Resumen}

La organización de los Testigos de Jehová es estudiada aqui a partir de los impresos que propone y que difunde masivamente, con el fin de proponer nuevas pistas de investigación sobre dicha organización. La participación en el movimiento de la Torre de la Guardia corresponde en efecto a un conjunto de prácticas sociales ligadas a los objetos impresos (fabricación en grandes cantidades, distribución puerta a puerta, lecturas individuales y en grupo). El examen de las relaciones sociales que se organizan alrededor de los impresos aporta también una luz nueva a la vez sobre la trayectoria histórica de la organización y sobre su funcionamiento presente. Desde esta perspectiva, los impresos aparecen como intermediarios que organizan y estructuran la experiencia " jehovista".

Palabra clave : Testigos de Jehová, prensa religiosa, organización religiosa, proselitismo. 